\title{
Labe㑾重要
}

Arquivos de Ciências do Mar

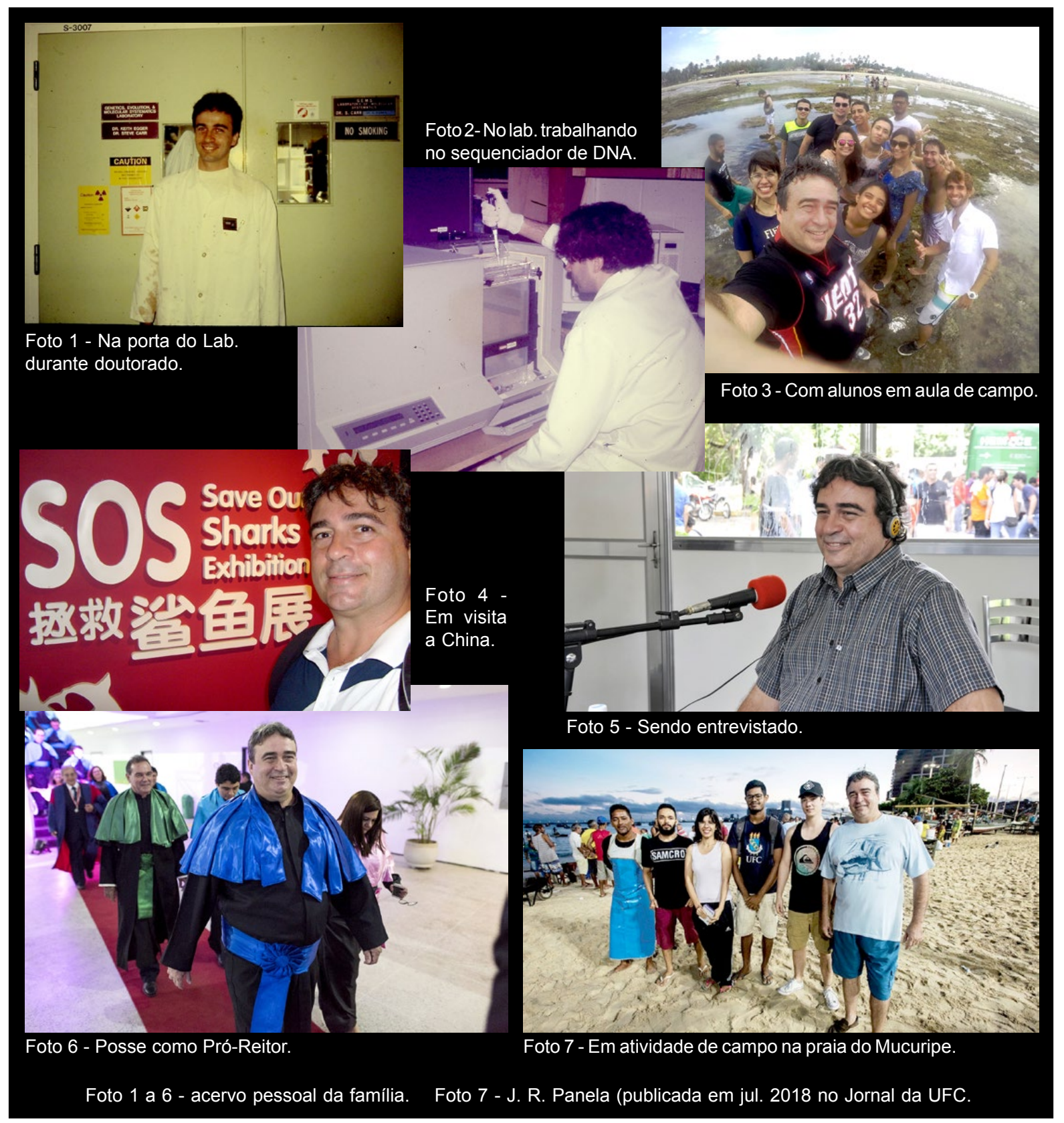

\section{MANUEL ANTONIO DE ANDRADE FURTADO NETO 1963 - 2019}

\section{Mayra Bezerra Vettorazzi ${ }^{1}$, Vicente Vieira Faria ${ }^{2}$}

Laboratório de Evolução e Conservação de Vertebrados Marinhos (EvolVe), Departamento de Biologia,

Centro de Ciências, Universidade Federal do Ceará, Campus do Pici.

URL: https://evolve.ufc.br/ - E-mails: mayra.vettorazzi@hotmail.com¹; vicentefaria@gmail.com² 


\title{
RESUMO
}

Manuel Antonio de Andrade Furtado Neto foi um pesquisador pioneiro no campo da genética populacional, da conservação e evolutiva de vertebrados marinhos no Brasil. Ele foi um dos primeiros cientistas no país a aplicar a, hoje famosa, técnica de reação em cadeia da polimerase (PCR) a organismos aquáticos. Ele forneceu, na década de 1990, a primeira evidência genética que sugeria que o boto-cinza (costa atlântica da América do Sul) e o tucuxi (bacia amazônica) eram espécies distintas. Na época, o boto-cinza e o tucuxi eram ainda considerados uma mesma espécie, Sotalia fluviatilis, e a divergência entre eles, ao nível de espécie, foi confirmada por outros colegas apenas na década seguinte. Também esteve na vanguarda da aplicação de técnicas de marcadores moleculares a outros organismos marinhos do país, incluindo pargos, atuns, lagostas e tubarões. Entre estes, o estudo da filogenia molecular dos cações-anjo se destaca, tendo sido, até o momento, o único a gerar e disponibilizar publicamente uma sequência de DNA da espécie de cação-anjo criticamente ameaçada Squatina argentina.

Palavras-chave: obituário, engenharia de pesca, DNA mitocondrial, golfinho, tubarão, administração acadêmica, administração do estado (pública).

\begin{abstract}
Manuel Antonio de Andrade Furtado Neto was a pioneer researcher in the field of population, conservation, and evolutionary genetics of marine vertebrates in Brazil. He was one of the first scientists in the country to apply the now-famous polymerase chain reaction (PCR) technique to aquatic organisms. He provided, in the 1990s, the first genetic evidence to suggest that the Guiana Dolphin (South American Atlantic Coast) and the Tucuxi (Amazon Basin) were distinct species. At that time, Guiana and Tucuxi dolphins were still considered a single species, Sotalia fluviatilis the species level divergence between them was only confirmed by other colleagues in the following decade. He was also at the forefront of applying techniques of molecular markers to other marine organisms in the country, including snappers, tuna, lobsters and sharks. Among these, the study of the molecular phylogeny of angelsharks stands out, having been, until now, the only study to generate and make publicly available a DNA sequence of the Critically Endangered Argentine Angelshark, Squatina argentina.
\end{abstract}

Keywords: obituary, fisheries engineering, mitochondrial DNA, dolphin, shark, academic administration, state (public) administration.

Em 14 de dezembro de 2019, a comunidade científica recebeu, com pesar, a morte prematura do Dr. Manuel Antonio de Andrade Furtado Neto, professor associado do Departamento de Engenharia de Pesca, da Universidade Federal do Ceará (UFC), aos 56 anos de idade, após batalha contra um linfoma. Manuel foi pioneiro na pesquisa com tubarões e raias, tendo sido um dos fundadores, em 1985, do Grupo de Trabalho sobre 
Pesca e Pesquisa de Tubarões e Raias no Brasil, que foi o embrião da Sociedade Brasileira para Estudos de Elasmobrânquios (SBEEL). Reconhecido internacionalmente, fez parte do Grupo de Especialistas de Tubarões (SSG), da União Internacional para a Conservação da Natureza (IUCN). Ele representou o Brasil em encontros da Organização das Nações Unidas (ONU) sobre diferentes temáticas ligadas a recursos aquáticos e desenvolvimento sustentável. Por seu brilhantismo, foi condecorado diversas vezes, local, nacional e internacionalmente.

Manuel Furtado Neto nasceu em Fortaleza, capital do Ceará, em 4 de outubro de 1963. Neto do primeiro vice-reitor da UFC e filho da primeira mulher a se formar engenheira civil pela UFC e que se tornaria, posteriormente, também a primeira professora do curso de Engenharia Civil dessa mesma universidade, Manuel sempre esteve envolvido em círculos acadêmicos. Segundo relato próprio, ele sempre nutriu grande curiosidade "sobre o que havia sob as águas do mar". Em 1981, foi aprovado em primeiro lugar para o curso de graduação em Engenharia de Pesca da UFC. O interesse particular nato se transformaria em objeto de dedicação profissional por toda a sua vida.

Começou a se destacar na carreira ainda estudante de graduação, tendo sido escolhido, entre graduandos de todo o país, como um dos 10 participantes do "Programa de Viagem de Intercâmbio Cultural de Jovens entre Brasil e Japão" (Youth Goodwill Mission), organizado pela Sede de Desenvolvimento do Gabinete do Primeiro Ministro do Japão. Retornando do Japão, ele estagiou em outras universidades do país, com destaque para a Universidade Federal do Rio Grande (FURG/RS), onde despertou o interesse pela pesca sustentável e conservação de tubarões. Ao retornar à UFC, decidiu então realizar sua pesquisa de trabalho de conclusão de curso com tubarões. Já um engenheiro de pesca, obteve uma bolsa de pesquisa em bioquímica de lagostas no Instituto de Ciências do Mar (Labomar) da UFC. Isso lhe abriu os olhos para a aplicação de ferramentas moleculares ao estudo de organismos marinhos, o que o levou a nova aprovação em primeiro lugar em uma seleção, desta vez no mestrado em Bioquímica na UFC. Lá, além da pesquisa, ele iniciou sua experiência didática no exercício do magistério superior, tornando-se professor substituto do Departamento de Biologia. Mas foi durante os anos de doutorado em Biologia na Memorial University of Newfoundland, no Canadá, que as maiores contribuições científicas do Manuel vieram à tona, tais como as pesquisas envolvendo o estudo do DNA de golfinhos e cações-anjo, além de pesquisas voltadas para baleias, pargos e atuns, dando-lhe reconhecimento internacional.

De volta ao Brasil em 1998, ele atuou novamente como professor substituto no Departamento de Biologia da UFC. No ano seguinte, ingressou como professor efetivo no Departamento de Engenharia de Pesca da UFC e fundou o Grupo de Estudos de Elasmobrânquios do Ceará (Elace). Esse grupo tem por objetivo fomentar a capacitação de pessoal para desenvolver pesquisas morfológicas, ecológicas, de dinâmica populacional e de biologia pesqueira de tubarões e raias, bem como realizar cursos em geral, promover eventos na área e fornecer assistência técnico-científica a comunidades pesqueiras litorâneas. Participou ainda do Grupo de Ictiologia Marinha Tropical (IMAT). Além disso, Manuel participou ativamente da pesquisa, da orientação de alunos e da produção científica do Grupo de Estudos de Cetáceos do Ceará (GECC), tendo sido um de seus fundadores e coordenador de projeto. O GECC posteriormente se transformou no Programa de Mamíferos Marinhos da bem-sucedida e prestigiada ONG Associação de Pesquisa e Preservação de Ecossistemas Aquáticos - Aquasis. 
Em 2003, assumiu a coordenação do Programa de Pós-Graduação em Engenharia de Pesca da UFC, tendo participado ativamente da implementação do doutorado no programa. Em 2008, assumiu a direção do Instituto de Ciências do Mar (Labomar/UFC), cargo no qual permaneceu até 2011. Durante esse tempo, atuou na criação do curso de graduação em Oceanografia da UFC, sendo então seu primeiro coordenador. Ele também participou da criação do curso de graduação em Ciências Ambientais. Como gestor, teve outras ocupações de destaque na comunidade cearense. Foi secretário adjunto do Ceará, na Secretaria de Pesca e Aquicultura, entre 2011 e 2014, exercendo papel central na área de políticas públicas para a gestão de recursos pesqueiros e aquícolas do estado. Finalmente, entre 2015 e 2019, foi Pró-Reitor de Assuntos Estudantis da UFC. Durante esse período em que esteve diretamente envolvido em atividades administrativas, ele ainda realizou pesquisas em piscicultura marinha e foi pioneiro também na aplicação de biotecnologia na área, além de orientar alunos de graduação e pós-graduação e supervisionar pesquisadores-doutores.

Devotado às Ciências do Mar, Manuel Furtado Neto demonstrava grande empolgação quanto ao ato de ensinar, tendo lecionado disciplinas para alunos dos cursos de graduação em Engenharia de Pesca, Ciências Biológicas, Oceanografia, Gestão de Políticas Públicas, entre outros cursos das áreas da Saúde e Agrárias. Também lecionou nos Programas de Pós-Graduação em Engenharia de Pesca e em Ciências Marinhas Tropicais. Sua empolgação e alegria se estendiam também a todos os desdobramentos que seu trabalho trazia aos alunos, à universidade e à comunidade em geral, sem nunca deixar de lado a simplicidade. Sempre atento às necessidades dos alunos, ele demonstrava maestria na lida dos assuntos profissionais e pessoais de todos a quem tocou. Após defesas de teses, em congressos ou ao receber prêmios, Manuel Furtado Neto, sempre expansivo, confraternizava calorosamente com seus pares, alunos, ex-alunos e familiares. Essas reuniões eram extremamente divertidas e polvilhadas de assuntos diversos, especialmente futebol, paixão indelével em todos os momentos.

Gerações de profissionais e cientistas marinhos brasileiros têm nele grande expoente e mestre em sentido amplo e literal. A bem da verdade, ficamos com o seu modelo de dedicação à ciência, liderança, alto astral e com muitas saudades. Seu legado de lealdade, amizade e incansável força de vontade continuará através de seus filhos Bárbara, Nadine, Ana Maia e André e de sua esposa Ana Caroline M. V. de Andrade Furtado (Carol). 


\section{TESE DE DOUTORADO}

Furtado Neto, A.A. Molecular systematics and population genetics of marine vertebrates from Brazil. 1998. Tese de doutorado, Biology, Memorial University of Newfoundland (Canadá), 188 p. Disponível em: http://www.repositorio.ufc.br/handle/riufc/33.

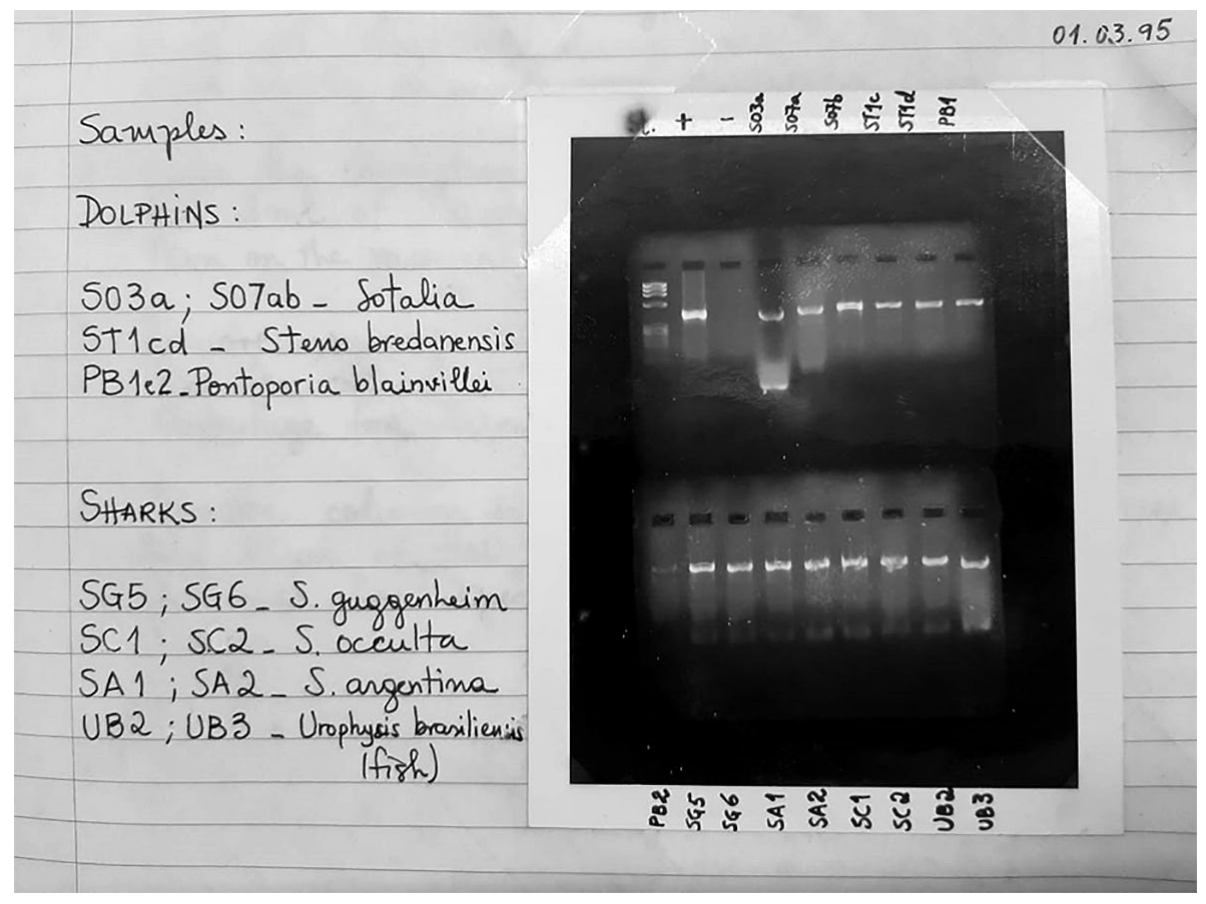

Trecho de um dos cadernos de laboratório utilizados pelo Manuel A. A. Furtado Neto durante sua pesquisa de doutorado em Biologia na Memorial University of Newfoundland, no Canadá. À esquerda, os códigos que identificam as amostras de tecido de golfinhos, cações-anjo e um peixe ósseo que tiveram seu DNA extraído e incluído em um experimento de reação em cadeia da polimerase (PCR) para amplificação (cópia) de um trecho do genoma mitocondrial. À direita, imagem obtida por visualização de gel revelando o resultado da PCR.

\section{Capítulos da tese escritos em formato de artigo}

Mitochondrial DNA sequence variation in the Brazilian dolphin Sotalia fluviatilis from coastal waters. Capítulo 2, p. 20-39.

Molecular phylogeny of cetaceans as suggested by the cytochrome oxidase I gene alone and in combination with other mitochondrial genes. Capítulo 3, p. 40-96.

Phylogenetic relationships of Angel sharks (Squatinidae, Elasmobranchii) from Southern Brazil as suggested by the mitochondrial cytochrome b gene. Capítulo 4, p. 97-119.

Genetic analysis of the stock structure of the Red snapper, Lutjanus purpureus, in Northen Brazil. Capítulo 5, p. 120-135.

Genetic analysis of the stock structure of the Yellowfin tuna, Thunnus albacares, in the southwest equatorial Atlantic Ocean. Capítulo 6, p. 136-151. 


\section{ARTIGOS CIENTÍFICOS}

1. Oliveira, J.A.; Ávila, F.J.C.; Alves Júnior, T.T.; Furtado Neto, M.A.A. \& Monteiro Neto, C. Monitoramento do boto cinza, Sotalia fluviatilis (Cetacea, Delphinidae) em Fortaleza, estado do Ceará, Brasil. Arquivos de Ciências do Mar, v. 29, p. 28-35, 1995.

2. Alves Júnior, T.T.; Ávila, F.J.C.; Oliveira, J.A.; Furtado Neto, M.A.A. \& Monteiro Neto, C. Registros de cetáceos para o litoral do estado do Ceará, Brasil. Arquivos de Ciências do Mar, v. 30, p. 79-92, 1996.

3. Gadig, O.B.F.; Bezerra, M.A. \& Furtado Neto, M.A.A. Novos registros e dados biológicos do tubarão-gato Schroederichthys tenuis Springer, 1966 (Chondrichthyes, Scyliorhinidae) para a costa norte do Brasil. Revista Nordestina de Biologia, v. 11, p. 51$55,1996$.

4. Gadig, O.B.F.; Bezerra, M.A. \& Furtado Neto, M.A.A. Nota sobre a biologia do tubarão Carcharhinus perezi (Poey, 1861) (Chondrichthyes, Carcharhinidae) do Norte-Nordeste do Brasil. Revista Nordestina de Biologia, v. 11, p. 31-36, 1996.

5. Furtado Neto, M.A.A.; Queiroz, E.L.; Zerbini, A.N. \& Carr, S.M. Uso de sequências do mtDNA para identificação de um exemplar de golfinho rotator, Stenella longirostris (Gray, 1828) encalhado no estado da Bahia, Brasil. Arquivos de Ciências do Mar, v. 31, p. 83-91, 1998.

6. Gadig, O.B.F.; Medina, A.; Bezerra, M.A. \& Furtado Neto, M.A.A. Dados sobre Squatina dumeril (Chondrichthyes, Squatinidae) com comentários taxonômicos do gênero Squatina em águas brasileiras. Arquivos de Ciências do Mar, v. 32, p. 133-136, 1999.

7. Parente, C.L.; Furtado Neto, M.A.A.; Alves, M.I.M. \& Monteiro Neto, C. Estudo da morfologia dos ossos tímpano-perióticos de cetáceos da sub-ordem Odontoceti (Mammalia, Cetacea). Arquivos de Ciências do Mar, v. 32, p. 103-110, 1999.

8. Gadig, O.B.F.; Bezerra, M.A.; Feitosa, R.D. \& Furtado Neto, M.A.A. Ictiofauna marinha do estado do Ceará: I. Elasmobranchii. Arquivos de Ciências do Mar, v. 33, p. 127-132, 2000.

9. Vieira, V.L.A.; Eldeir, A.C.A.; Furtado Neto, M.A.A. \& Carr, S.M. Genetic analysis of the stock structure of the yellowfin tuna, Thunnus albacares, in the Southwest Equatorial Atlantic Ocean as suggested by mitochondrial DNA sequences. Arquivos de Ciências do Mar, v. 33, p. 119-126, 2000.

10. Monteiro Neto, C.; Alves Júnior, T.T.; Ávila, F.J.C.; Campos, A.A.; Costa, A.F.; Silva, C.P.N. \& Furtado Neto, M.A.A. Impact of fisheries on the tucuxi (Sotalia fluviatilis) and rough-toothed dolphin (Steno bredanensis) populations off Ceará State, northeastern Brazil. Aquatic Mammals, v. 26, p. 49-56, 2000.

11. Furtado Neto, M.A.A. \& Monteiro Neto, C. Preservação de mamíferos marinhos do Nordeste. Ciência Animal, 10 (suplemento 1, número 1, publicado como Anais do II Simpósio Cearense de Ciência Animal), p. 48-51, 2000.

12. Silva, G.B.; Viana, M.S.R. \& Furtado Neto, M.A.A. Morfologia e alimentação da raia Dasyatis guttata (Dasyatidae; Elasmobranchii) na enseada do Mucuripe, Fortaleza, Ceará. Arquivos de Ciências do Mar, v. 34, p. 67-75, 2001. 
13. Correia, L.B.; Feitosa, R.D. \& Furtado Neto, M.A.A. Monitoramento de tubarões marcados e devolvidos ao mar no Parque Estadual Marinho Pedra da Risca do Meio, estado do Ceará. Ciência Animal, 12 (suplemento 1, número 1, publicado como Anais do IV Simpósio Cearense de Ciência Animal e II Simpósio Cearense de Buiatria), p. 108-111, 2002.

14. Furtado Neto, M.A.A. \& Carr, S.M. Molecular genetics of some Brazilian sharks. Shark News - Newsletter of the IUCN Shark Specialist Group, 14, p. 10, 2002.

15. Gurjão, L.M.; Furtado Neto, M.A.A.; Santos, R.A. \& Cascon, P. Feeding habits of marine tucuxi, Sotalia fluviatilis, at Ceará state, Northeastern Brazil. The Latin American Journal of Aquatic Mammals, v. 2, p. 117-122, 2003.

16. Gurjão, L.M.; Furtado Neto, M.A.A.; Santos, R.A. \& Cascon, P. Notas sobre a dieta de cachalotes (Cetacea: Physeteroidea), encalhados no Ceará, Nordeste do Brasil. Arquivos de Ciências do Mar, v. 36, p. 67-76, 2003.

17. Monteiro Neto, C.; Vila, F.J.C.A.; Alves Jr., T.T.; Araújo, D.S.; Campos, A.A.; Martins, A.M.A.; Parente, C.L.; Furtado Neto, M.A.A. \& Lien, J. Behavioral responses of Sotalia fluviatilis (Cetacea, Delphinidae) to acoustic pingers, Fortaleza, Brazil. Marine Mammal Science, 20, p. 145-151, 2004.

18. Martins, A.M.; Alves Júnior, T.T.; Furtado Neto, M.A.A. \& Lien, J. The most northern record of Gervais beaked whale, Mesoplodon europaeus (Gervais, 1855), for the Southern Hemisphere. The Latin American Journal of Aquatic Mammals, 3, p. 151-155, 2004.

19. Gurjão, L.M.; Furtado Neto, M.A.A.; Santos, R.A. \& Cascon, P. Análise de conteúdos estomacais de quatro golfinhos (Cetacea: Delphinidae) encalhados em praias no litoral do estado do Ceará, Brasil. Revista Biociências, v. 10, p. 39-45, 2004.

20. Meirelles, A.C.O. \& Furtado Neto, M.A.A. Stranding of an Antarctic minke whale, Balaenoptera bonaerensis Burmeister, 1867, in the Northern coast of South America. The Latin American Journal of Aquatic Mammals, 3, p. 81-82, 2004.

21. Osório, F.M.; Marinho, R.A.; Lotufo, T.M.C. \& Furtado Neto, M.A.A. Primeiro registro de Pomadasys ramosus Poey, 1860 (Perciformes: Haemulidae) para a costa do Ceará, Brasil. Arquivos de Ciências do Mar, v. 38, p. 127-130, 2005.

22. Gonçalves, M.M.; Lemos, M.V.F.; Galetti Junior, P.M.; Freitas, P.D. \& Furtado Neto, M.A.A. Fluorescent amplified fragment length polymorphism (fAFLP) analyses and genetic diversity in Litopenaeus vannamei (Penaeidae). Genetics and Molecular Biology, v. 28, p. 267-270, 2005.

23. Furtado Neto, M.A.A. \& Barros Júnior, F.V.P. Análise da produção pesqueira de elasmobrânquios no estado do Ceará, Brasil, de 1991 a 2003. Arquivos de Ciências do Mar, v. 39, p. 110-116, 2006.

24. Vaz, L.A.L.; Carreiro, C.R.P.; Goulart-Filho, L.R.; Furtado Neto, M.A.A. (2006). Relações filogenéticas em raias (Dasyatis, Elasmobranchii) do estado do Ceará, Brasil. Arquivos de Ciências do Mar, v. 39, p. 86-88, 2006.

25. Salles, R.; Fonteles Filho, A.A.; Furtado Neto, M.A.A.; Carr, S.M. \& Freitas, S.M. Morphometric and mitochondrial DNA analyses of the Caribbean red snapper, Lutjanus 
purpureus (Teleostei, Lutjanidae), in Western Atlantic off Northern Brazil. Boletim do Instituto de Pesca, v. 32, p. 115-125, 2006.

26. Borges, D.A.; Bezerra, M.A. \& Furtado-Neto, M.A.A. Avaliação do ponto de equilíbrio e lucratividade aplicados a sistemas de produção de camarão marinho, no estado do Ceará, Brasil. Arquivos de Ciências do Mar, v. 40, n. 2, p. 42-46, 2007.

27. Nascimento, A.R.; Carvalho, E.P.; Furtado Neto, M.A.A.; Martins, A.G.L.A. \& Vieira, R.H.S.F. Atividade antibacteriana de óleos essenciais frente a bactérias isoladas de sururu Mytella falcata. Arquivos de Ciências do Mar, v. 40, n. 2, p. 47-54, 2007.

28. Santander Neto, J.; Jucá-Queiroz, B.; Nascimento, F.C.P.; Basílio, T.H.; Medeiros, R.S.; Furtado Neto, M.A.A. \& Faria, V.V. On the occurence of sevengill and sixgill sharks (Hexanchiformes: Hexanchidae) off Ceará State, Brazil, Western Equatorial Atlantic. Arquivos de Ciências do Mar, v. 40, n. 2, p. 59-63, 2007.

29. Furtado Neto, M.A.A.; Marinho, R.A.; Conceição, R.N.L.; Salles, R.; Braga, M.S.C.; Leite Filho, R.H.; Feitosa, R.D.; Basílio, T.H.; Nascimento, F.C.P.; Osório, F.M.; Fonteles Filho, A.A. \& Maia, L.P. Barco escola: transferência de tecnologia e capacitação de pescadores artesanais para a captura de peixes durante o defeso da lagosta no Nordeste do Brasil. Arquivos de Ciências do Mar, v. 40, n. 2, p. 75-79, 2007.

30. Basílio, T.H.; Faria, V.V. \& Furtado Neto, M.A.A. Fauna de elasmobrânquios do estuário do rio Curu, Ceará, Brasil. Arquivos de Ciências do Mar, v. 41, n. 2, p. 65-72, 2008.

31. Jucá-Queiroz, B.; Santander Neto, J.; Medeiros, R.S.; Nascimento, F.C.P.; Furtado Neto, M.A.A.; Faria, V.V. \& Rincon, G. Cartilaginous fishes (Class Chondrichthyes) off Ceará State, Brazil, western equatorial Atlantic - an update. Arquivos de Ciências do Mar, v. 41, n. 2, p. 73-81, 2008.

32. Feijó, R.G.; Kamimura, M.T.; Oliveira, D.M.; Costa, R.B.; Furtado Neto, M.A.A.; Coelho, M.G.L. \& Maggioni, R. Avaliação da variabilidade genética do vírus da Infecção Hipodermal e Necrose Hematopoiética (IHHNV) no camarão rosa, Farfantepenaeus subtilis (Pérez-Farfante, 1967). Arquivos de Ciências do Mar, v. 41, n. 1, p. 113-117, 2008.

33. Leite Filho, R.H.; Marinho, R.A. \& Furtado Neto, M.A.A. Capacitação de pescadores artesanais e cooperativismo no Ceará: lições do Projeto Barco-Escola. Arquivos de Ciências do Mar, v. 41, n. 1, p. 123-127, 2008.

34. Basílio, T.H.; Godinho, W.O.; Araújo, M.E.; Furtado Neto, M.A.A. \& Faria, V. V. Ictiofauna do estuario do rio Curu, Ceará, Brasil. Arquivos de Ciências do Mar, v. 42, n. 2, p. 81-88, 2009.

35. Faria, V.V.; Venancio, I.M.; Basílio, T.H.; Silveira, L.M.; Jucá-Queiroz, B.; Gadig, O.B.F. \& Furtado Neto, M.A.A. Captura incidental de um tubarão-baleia, Rhincodon typus (Orectolobiformes, Rhincodontidae), na costa do Ceará, Nordeste do Brasil. PanAmerican Journal of Aquatic Sciences, v. 4, n. 4, p. 599-604, 2009.

36. Santos, E.S.; Furtado Neto, M.A.A.; Mota, S.; Santos, A.B. \& Aquino, M.D. Cultivo de tilápia do Nilo em esgoto doméstico tratado, para diferentes taxas de alimentação. Revista DAE, v. 180, p. 4-11, 2009.

37. Vettorazzi, M.B.; Teixeira, E.G.; Souza, R.L.M.; Cesar, J.R.O. \& Furtado Neto, M.A.A. Motilidade espermática do sêmen do Ariacó, Lutjanus synagris. Arquivos de Ciências do Mar, v. 43, n. 2, p. 21-26, 2010. 
38. Costa, J.M.; Moreira, R.L.; Fonteles Filho, A.A.; Cavalcante, P.P.L. \& Furtado Neto, M.A.A. Caracterização e viabilidade socioeconômica da pesca de lagosta viva na praia da Caponga, Cascavel, estado do Ceará. Arquivos de Ciências do Mar, v. 44, n. 2, p. 29-35, 2011.

39. Santander Neto, J.; Shinozaki-Mendes, R.A.; Silveira, L.M.; Jucá-Queiroz, B.; Furtado Neto, M.A.A. \& Faria, V.V. Population structure of nurse sharks, Ginglymostoma cirratum (Orectolobiformes), caught off Ceará State, Brazil, south-western Equatorial Atlantic. Journal of the Marine Biological Association of the United Kingdom, v. 91, n. 6, p. 1193-1196, 2011.

40. Jucá-Queiroz, B.; Silva, A.C.; Nascimento, F.C.P. \& Furtado Neto, M.A.A. Composição e abundância de elasmobrânquios capturados com espinhel de fundo no litoral oeste do estado do Ceará. Arquivos de Ciências do Mar, v. 44, n. 2, p. 5-11, 2011.

41. Carreiro, C.R.P.; Furtado Neto, M.A.A.; Mesquita, P.E.C. \& Bezerra, T. Sex determination in the giant fish of Amazon Basin, Arapaima gigas (Osteoglossiformes, Arapaimatidae), using laparoscopy. Acta Amazonica, v. 41, n. 3, p. 415-420, 2011.

42. Cavalcante, P.P.L. \& Furtado Neto, M.A.A. Implementação de quotas individuais transferíveis e do desembarque compulsório de lagosta viva como estratégia de ordenamento da pesca. Arquivos de Ciências do Mar, v. 45, n. 2, p. 49-59, 2012.

43. Faria, V.V.; Rolim, L.S.; Vaz, L.A.L. \& Furtado Neto, M.A.A. Reevaluation of RAPD markers involved in a case of stingray misidentification (Dasyatidae: Dasyatis). Genetics and Molecular Research, v. 11, n. 4, p. 3835-3845, 2012.

44. Bezerra, M.A.; Furtado Neto, M.A.A. \& Fonteles Filho, A.A. Experimentos sobre a chance de escape de lagostas do gênero Panulirus, capturadas por cangalha. Arquivos de Ciências do Mar, v. 46, n. 2, p. 5-12, 2013.

45. Falcão, L.H.O.; Furtado Neto, M.A.A.; Maggioni, R. \& Faria, V.V. Prospective molecular markers for the identification of illegally traded angelsharks (Squatina) and dolphin (Sotalia guianensis). Genetics and Molecular Research, v. 13, p. 9710-9717, 2014.

46. Holanda, P.C.; Furtado Neto, M.A.A. \& Cabral, N.R.A.J. An evaluation of public aquariums in São Paulo (Brazil) in light of the Global Aquarium Strategy for Conservation and Sustainability. Arquivos de Ciências do Mar, v. 48, p. 5-15, 2015.

47. Souza, R.L.M.; Vettorazzi, M.B.; Kobayashi, R.K. \& Furtado Neto, M.A.A. Eugenol como anestésico no manejo de ariacó, Lutjanus synagris (Linnaeus, 1758), cultivado. Revista Ciência Agronômica, v. 46, p. 532-538, 2015.

48. Bezerra, M.A.; Lustosa, D.C.P. \& Furtado Neto, M.A.A. Padrões hidrobiológicos como indicadores ambientais em águas afluentes e efluentes de viveiros de carcinicultura marinha no estado do Ceará. Revista Brasileira de Ciências Ambientais, v. 41, p. 75-85, 2016.

49. Lustosa Neto, A.D.; Nunes, M.L.; Ferreira, R.N.C.; Bezerra, J.H.C. \& Furtado Neto, M.A.A. Elaboração, rendimento e custo de almôndegas de tilápia do Nilo e pirarucu cultivados: aplicação na merenda escolar. Acta of Fisheries and Aquatic Resources, v. 4, n. 2, p. 101-109, 2016. 
50. Souza, R.L.M.; Vettorazzi, M.B.; Kobayashi, R.K. \& Furtado Neto, M.A.A. Reprodução induzida de ariacó, Lutjanus synagris (Linnaeus, 1758) sob cultivo, em resposta à indução hormonal. Arquivos de Ciências do Mar, v. 49, n. 2, p. 68-76, 2016.

51. Castro, L.A.; Masih Neto, T.; Paulo, E.A. \& Furtado Neto, M.A.A. Avaliação do rendimento dos filés e resíduos da filetagem do beijupirá cultivado e selvagem. Arquivos de Ciências do Mar, v. 50, n. 1, p. 15-24, 2017.

52. Falcão, L.H.O.; Campos, A.S.; Freitas, J.E.P.; Furtado Neto, M.A.A. \& Faria, V.V. Molecular identification of cetaceans from the West Atlantic using the E3-I5 region of COI. Genetics and Molecular Research, v. 16, p. gmr16029607, 2017.

53. Lustosa Neto, A.D.; Nunes, M.L.; Maia, L.P.; Barbosa, J.M.; Lira, P.P. \& Furtado Neto, M.A.A. Almôndegas de pirarucu e tilápia nilótica: caracterização e aplicação na merenda escolar. Acta of Fisheries and Aquatic Resources, v. 6, n. 2, p. 1-12, 2018.

54. Lustosa Neto, A.D.; Nunes, M.L.; Maia, L.P.; Bezerra, J.H.C.; Barbosa, J.M.; Lira, P.P. \& Furtado Neto, M.A.A. A indústria de produtos derivados da pesca e aquicultura. Acta of Fisheries and Aquatic Resources, v. 6, n. 2, p. 28-48, 2018.

\section{ORIENTAÇÃO DE ESTUDANTES}

\section{A) Orientação na graduação (estágio supervisionado e monografia / trabalho de conclusão de curso)}

1. Douglas Silva Araújo - relatório de estágio supervisionado, 2000. Relatório de estágio em aplicação de técnicas de fotoidentificação de pequenos cetáceos, realizado junto à sede da Coalisão Internacional da Vida Silvestre (IWC/Brasil), em Florianópolis, Santa Catarina. Engenharia de Pesca, Universidade Federal do Ceará (UFC).

2. Guelson Batista da Silva - monografia, 2001. Morfologia e alimentação de Dasyatis guttata (Dasyatidae; Elasmobranchii), na enseada do Mucuripe, Fortaleza, Ceará. Engenharia de Pesca, UFC.

3. Rommel Darlan Feitosa - monografia, 2002. Diagnóstico da pesca e aquicultura no município de Icapuí: subsídios para o ordenamento pesqueiro e aquícola. Engenharia de Pesca, UFC.

4. Ana Carolina Oliveira de Meirelles - monografia, 2003. Diagnóstico de mortalidade de peixe-boi marinho Trichechus manatus manatus Linnaeus, 1758, no litoral leste do estado do Ceará. Ciências Biológicas, UFC.

5. Luíza Janaína Lopes Barroso Pinto - relatório de estágio supervisionado, 2003. Acompanhamento do controle de qualidade durante o beneficiamento industrial do camarão para exportação. Engenharia de Pesca, UFC.

6. Francisco José Capibaribe Ávila - monografia, 2003. Testes acústicos com alarmes no comportamento de botos da espécie Sotalia fluviatilis na Praia de Iracema, FortalezaCeará. Ciências Biológicas, UFC.

7. Helen Maria Duarte do Rego Barros - monografia, 2003. Registros de encalhes de cachalotes, Physeter macrocephalus, na costa do estado do estado do Ceará. 2003. Ciências Biológicas, UFC. 
8. Cristiano Teixeira Gomes - relatório de estágio supervisionado, 2003. Relatório de estágio na larvicultura e engorda em uma fazenda de camarão marinho. Engenharia de Pesca, UFC.

9. Ana Karla Correia da Silva - relatório de estágio supervisionado, 2003. Relatório de estágio no setor de beneficiamento de camarão da empresa de pescado Ipesca. Engenharia de Pesca, UFC.

10. Francisco Valmir Paula Barros Júnior - monografia, 2004. Análise da produção de pesca de elasmobrânquios no estado do Ceará. Engenharia de Pesca, UFC.

11. Nayra Carmem Silva Cavalcante - monografia, 2004. Levantamento sobre as ações da Secretaria Especial de Aquicultura e Pesca da Presidência da República (SEAP/PR). Engenharia de Pesca, UFC.

12. Lúcia de Fátima de Souza e Silva - relatório de estágio supervisionado, 2004. Acompanhamento de transporte de tambaqui vivo (Colossoma macropomum). Engenharia de Pesca, UFC.

13. Mônica Rego Maciel - monografia, 2004. Povoamento dos açudes públicos no estado do Ceará: manejo, educação e pesquisa. Engenharia de Pesca, UFC.

14. Aline Maria Araújo Martins - monografia, 2004. Análise dos registros de cetáceos para o litoral do estado do Ceará, 1992-2003. Ciências Biológicas, UFC.

15. Artur Cláudio Alves Batista - relatório de estágio supervisionado, 2004. Acompanhamento das atividades de produção em uma fazenda de cultivo de camarão marinho. Engenharia de Pesca, UFC.

16. Rebeka Alynne Moreira - relatório de estágio supervisionado, 2004. Acompanhamento do controle de qualidade durante o beneficiamento industrial do camarão na empresa COMINALLI. Engenharia de Pesca, UFC.

17. Jacinta de Fátima Souza e Silva - relatório de estágio supervisionado, 2004. Beneficiamento e processamento tecnológico da tilápia do Nilo (Oreochromis niloticus var. chitralada). Engenharia de Pesca, UFC.

18. Marleon Alves Damasceno - monografia, 2004. Monitoramento do boto cinza, Sotalia fluviatilis (Gervais, 1853), na praia de Iracema, Fortaleza (CE), entre os anos de 1992 a 2000. Engenharia de Pesca, UFC.

19. Wesley Felix Batista - monografia, 2004. Levantamento da ictiofauna no afluente de uma fazenda de camarão: composição, distribuição e aspectos bioecológicos. Engenharia de Pesca, UFC.

20. Thalma Clara Escócia da Silva - relatório de estágio supervisionado, 2005. Acompanhamento das atividades desenvolvidas no Centro de Pesquisas em Aquicultura Rodolpho von Ihering do DNOCS, em Pentecoste, Ceará. Engenharia de Pesca, UFC.

21. Antonio Cesar Rodrigues Garcia - relatório de estágio supervisionado, 2005. Acompanhamento do processo de despesca do camarão HOSO (head on shell on) para o mercado externo, no município de Acaraú, Ceará. Engenharia de Pesca, UFC.

22. Andréa Soares Marinho - relatório de estágio supervisionado, 2005. Acompanhamento do processamento de peixes na indústria de pesca IPECEA. Engenharia de Pesca, UFC. 
23. Adail Oliveira de Sousa Júnior - relatório de estágio supervisionado, 2005. Acompanhamento do processo de engorda do camarão Litopenaeus vannamei cultivado na fazenda Cemar, em Paraipaba, estado do Ceará. Engenharia de Pesca, UFC.

24. Igor Melo de Pinho - relatório de estágio supervisionado, 2006. Acompanhamento do manejo alimentar de juvenis de cioba, Lutjanus analis, cultivados em laboratório. Engenharia de Pesca, UFC.

25. Rui Jorge de Carvalho - monografia, 2006. Levantamento faunístico de elasmobrânquios nos estuários do estado do Ceará. Engenharia de Pesca, UFC.

26. Daniela Accioly de Carvalho - relatório de estágio supervisionado, 2006. Acompanhamento no setor de beneficiamento de camarão na empresa de pescado Interfrios. Engenharia de Pesca, UFC.

27. Rubens Galdino Feijó - monografia, 2007. Avaliação da variabilidade do Vírus da Infecção Hipodermal e Necrose Hematopoiética (IHHNV) no camarão rosa Farfantepenaeus subtilis (Pérez-Farfante, 1967) infectado experimentalmente. Engenharia de Pesca, UFC.

28. Ricardo Silveira de Medeiros - monografia, 2007. Morfometria de condrocrânio em elasmobrânquios do Norte e Nordeste do Brasil. 2007. Engenharia de Pesca, UFC.

29. Eliana Matos Ribeiro - monografia, 2007. Estudos preliminares para a identificação sexual do pirarucu, Arapaima gigas (Cuvier) (Teleostei, Osteoglossidae), utilizando marcadores moleculares do tipo RAPD. Engenharia de Pesca, UFC.

30. Rodrigo Pinto de Castro - relatório de estágio supervisionado, 2008. Acompanhamento da produção de tilápias (Oreochromis niloticus var. chitralada) em piscicultura do município de Itapiúna, Ceará. Engenharia de Pesca, UFC.

31. Gledson de Freitas Fernandes - monografia, 2008. Caracterização da pesca artesanal no distrito de Bitupitá, município de Barroquinha, Ceará. Engenharia de Pesca, UFC.

32. Anita Antunes Ribeiro - monografia, 2008. Monitoramento multiparamétrico da qualidade da água dos viveiros do Centro de Pesquisas em Aquicultura Rodolpho von Ihering. Engenharia de Pesca, UFC.

33. Jones Santander Neto - monografia, 2008. Estrutura da população de tubarões-lixa (Ginglymostoma cirratum) capturados pela frota artesanal da enseada do Mucuripe no estado do Ceará. Engenharia de Pesca, UFC.

34. Julianna Albuquerque Martins - relatório de estágio supervisionado, 2008. Acompanhamento do beneficiamento do camarão Litopenaeus vannamei na Indústria $R \& B$ Aquicultura Comércio, Exportação e Importação Ltda. Engenharia de Pesca, UFC.

35. Thiago Holanda Basílio - monografia, 2008. A pesca e os pescadores do estuário do rio Curu, Ceará. 2008. Engenharia de Pesca, UFC.

36. Bruno Jucá de Queiroz - monografia, 2009. Análise de pescarias com espinhel de fundo sobre a plataforma continental do Nordeste do Brasil. Engenharia de Pesca, UFC.

37. Marcelo Alves da Silva - monografia, 2009. Políticas públicas necessárias para o desenvolvimento da pesca artesanal no Distrito de Bitupitá, Ceará. Engenharia de Pesca, UFC.

38. Paulo Alberto Peixoto Ferreira - relatório de estágio supervisionado, 2010. Crescimento e terminação do camarão marinho, Litopenaeus vannamei, cultivado na Fazenda Pearce Furtado Aquicultura e Agricultura Ltda., Itarema-CE. Engenharia de Pesca, UFC. 
39. Micael Lincoln Cardoso Paz - monografia, 2010. Plano Local de Desenvolvimento da Maricultura - PLDM: estudo de caso do município de Icapuí, CE. Engenharia de Pesca, UFC.

40. Felinto Holanda Cavalcante Souza - monografia, 2010. Avaliação no desempenho zootécnico da tilápia do Nilo (Oreochromis niloticus) mediante a utilização de probiótico comercial nas fases de alevinagem e recria no cultivo em sistemas de tanquesrede. Engenharia de Pesca, UFC.

41. Jamile Mota da Costa - monografia, 2011. Cadeia produtiva de lagosta viva no estado do Ceará, Brasil. Engenharia de Pesca, UFC.

42. Thiago Gomes Varela - monografia, 2013. Identificação e caracterização das etapas do processo de exportação de peixes ornamentais no estado do Ceará, durante o período de 2009 a 2011. Engenharia de Pesca, UFC.

43. Luiz Fágner Ferreira Nogueira - monografia, 2013. Perfil socioeconômico dos pescadores cearenses. Engenharia de Pesca, UFC.

44. Vladimir Vinícius de Oliveira Holanda - relatório de estágio supervisionado, 2013. Monitoramento do processo de vacinação de tilápias do Nilo (Oreochromis niloticus) no Projeto Curupati-Peixe, açude Castanhão. Engenharia de Pesca, UFC.

45. Thiago Pereira Menezes - monografia, 2015. Análise e caracterização da pesca de elasmobrânquios no estado do Ceará, Brasil, de 1998 a 2007. Engenharia de Pesca, UFC.

46. Jarlan Silva de Lima - monografia, 2015. A pesca nos currais-de-pesca de Bitupitá, município de Barroquinha, Ceará. Engenharia de Pesca, UFC.

47. José Belquior Gonçalves Neto - monografia, 2015. Análise do desembarque de tubarões e raias na enseada do Mucuripe, Fortaleza, Ceará, no período de 2010 a 2011. Engenharia de Pesca, UFC.

48. Caio de Góes Loira Parada - monografia, 2017. Caracterização da pesca artesanal de Paracuru, Ceará. Engenharia de Pesca, UFC.

49. Larissa Liberalino Marques - relatório de estágio supervisionado, 2017. Cultivo intensivo de camarão marinho, Penaeus vannamei, realizado na fazenda Joli Aquicultura Ltda., Jaguaruana-Ceará. Engenharia de Pesca, UFC.

50. Edson Luiz Marques Duarte - relatório de estágio supervisionado, 2017. Acompanhamento e descrição do cultivo semi-intensivo de Penaeus vannamei na Fazenda Aquisa Aquicultura Samaria Ltda. Engenharia de Pesca, UFC.

51. Débora de Lima Leite - monografia, 2018. Impactos gerados pelo Porto do Pecém nas comunidades de pescadores no distrito de Pecém. Engenharia de Pesca, UFC.

52. Raphael Loreno Sousa Santos - monografia, 2019. Desembarques de tubarões e raias na enseada do Mucuripe, Fortaleza, Ceará, no período de 2015 a 2017. Engenharia de Pesca, UFC.

\section{B) Orientação na pós-graduação (mestrado e doutorado)}

1. Carlos Riedel Porto Carreiro - mestrado, 2001. Identificação de populações da lagosta vermelha, Panulirus argus, no Norte e Nordeste do Brasil usando marcadores moleculares do tipo RAPD. Programa de Pós-graduação (PPG) em Engenharia de Pesca, UFC. 
2. Francisco José Lopes Cajado - mestrado, 2004. Avaliação dos procedimentos de introdução de tilápias tailandesas (Oreochromis niloticus var. chitralada) no estado do Ceará. PPG em Engenharia de Pesca, UFC.

3. Michelle Mantovani Gonçalves - mestrado, 2004. Diversidade genética do camarão Litopenaeus vannamei cultivado no Nordeste do Brasil. PPG em Engenharia de Pesca, UFC.

4. Sérgio Alberto Apolinário Almeida - mestrado, 2005. Efeito do uso de substrato artificial na sobrevivência e crescimento do camarão branco Litopenaeus vannamei (PerezFarfante e Kesley 1997), cultivado em gaiolas com diferentes densidades de estocagem. PPG em Engenharia de Pesca, UFC.

5. Letícia de Almeida Leão Vaz - mestrado, 2005. Variabilidade genética em raias do gênero Dasyatis (Dasyatidae; Elasmobranchii) no Brasil. PPG em Engenharia de Pesca, UFC.

6. Kléber Biana Santiago - mestrado, 2006. Biologia reprodutiva do pacu Myleus micans (Lütken, 1875) (Characidae: Serrasalminae) do rio São Francisco, região de Três Marias, Minas Gerais. PPG em Engenharia de Pesca, UFC.

7. Wesley Felix Batista - mestrado, 2006. Fauna ictiológica do estuário do rio Coreaú (Ceará-Brasil): composição, distribuição e aspectos bioecológicos. PPG em Engenharia de Pesca, UFC.

8. Mauro Vinícius Dutra Girão - mestrado, 2007. Avaliação dos procedimentos de introdução do pirarucu (Arapaima gigas) na região Nordeste: retrospectiva histórica, situação atual e perspectivas futuras. PPG em Engenharia de Pesca, UFC.

9. Raimundo Hélio Leite Filho - mestrado, 2008. Transferência de tecnologia e capacitação de pescadores artesanais para a pesca oceânica de pequena escala: o Projeto Barcoescola e cooperativismo. PPG em Engenharia de Pesca, UFC.

10. Gláuber Gomes de Oliveira - mestrado, 2008. Políticas públicas para o desenvolvimento da aquicultura no Ceará: uma comparação dos governos do estado do Ceará entre 1998 e 2008. PPG em Engenharia de Pesca, UFC.

11. Emanuel Soares dos Santos - mestrado, 2008. Cultivo de tilápias em esgoto doméstico tratado com diferentes taxas de alimentação. PPG em Engenharia de Pesca, UFC.

12. Valter Braga de Souza Júnior - mestrado, 2009. Piscicultura em módulos associativos familiares no estado do Ceará. PPG em Engenharia de Pesca, UFC.

13. Oscar Pacheco Passos Neto - mestrado, 2010. Avaliação da desova induzida hormonalmente e qualidade dos ovos de ariacó, Lutjanus synagris (Linnaeus, 1758). PPG em Ciências Marinhas Tropicais, UFC.

14. Carlos Riedel Porto Carreiro - doutorado, 2012. Inovações tecnológicas na sexagem, manejo reprodutivo e crescimento do pirarucu, Arapaima gigas (Schinz,1822) (Actinopterygii, Arapaimidae) cultivado no Centro de Pesquisas em Aquicultura Rodolph von Ihering (CPA) do DNOCS, Pentecoste, Ceará. PPG em Engenharia de Pesca, UFC.

15. Rossi Lelis Muniz Souza - doutorado, 2012. Reprodução induzida de ariacó, Lutjanus synagris (Bloch, 1772), em cativeiro. PPG em Ciências Marinhas Tropicais, UFC. 
16. João Felipe Nogueira Matias - doutorado, 2012. Análise da sustentabilidade da aquicultura em águas de domínio da União, nos parques aquícolas do reservatório do Castanhão, estado do Ceará: estudo de caso. PPG em Engenharia de Pesca, UFC.

17. Marcelo Augusto Bezerra - doutorado, 2013. Contribuições para o aprimoramento de políticas públicas na cadeia produtiva da lagosta. PPG em Engenharia de Pesca, UFC.

18. Paulo Parente Lira Cavalcante - doutorado, 2014. Ordenamento da pesca de lagosta (Gênero: Panulirus) e a recuperação da sustentabilidade das pescarias no litoral do Brasil: uma proposta inovadora do ordenamento pesqueiro. PPG em Engenharia de Pesca, UFC.

19. Renato Teixeira Moreira - doutorado, 2015. Cultivo de Arthrospira platensis em meio orgânico: extração de polissacarídeos sulfatados e sua administração a camarões Penaeus vannamei. PPG em Engenharia de Pesca, UFC.

20. Clara de Melo Coe - mestrado, 2015. Aspectos da pesca e perfil socioeconômico dos pescadores de Bitupitá, município de Barroquinha, Ceará. PPG em Engenharia de Pesca, UFC.

21. Priscila Carvalho Holanda - doutorado, 2016. O papel dos aquários públicos no Antropoceno: uma avaliação da "estratégia global dos aquários para conservação e sustentabilidade". PPG em Ciências Marinhas Tropicais, UFC.

22. Antonio Diogo Lustosa Neto - doutorado, 2016. Elaboração e caracterização de almôndegas de tilápia do Nilo (Oreochromis niloticus) e Pirarucu (Arapaima gigas) cultivados: aplicação na merenda escolar. PPG em Engenharia de Pesca, UFC.

23. Gabriel de Mesquita Facundo - mestrado, 2016. Captura, aclimatação e manejo de reprodutores de lutjanídeos, Lutjanus sp., e a indução à reprodução em cativeiro do ariacó L. synagris. PPG em Ciências Marinhas Tropicais, UFC.

24. Márcio Alves Bezerra - doutorado, 2017. Indicadores de sustentabilidade na aquicultura brasileira: um estudo de caso da carcinicultura marinha no estado do Ceará. PPG em Ciências Marinhas Tropicais, UFC.

25. Antonio Glaydson Lima Moreira - doutorado, 2017. Respostas metabólicas e hematológicas de tilápias do Nilo, Oreochromis niloticus, alimentadas com ração suplementada com Arthrospira platensis, submetida a diferentes agentes estressores. PPG em Engenharia de Pesca, UFC.

26. Luciana Antônia Araújo de Castro - doutorado, 2017. Processamento dos cortes comerciais e aproveitamento dos resíduos de beijupirás (Rachycentron canadum) cultivados e selvagens. PPG em Ciências Marinhas Tropicais, UFC.

27. Caio Lívio Bezerra Holanda - mestrado, 2017. Cultivo da microalga Chaetoceros muelleri (Lemmermann, 1898) em efluente de carcinicultura e seu efeito na qualidade de água, produção de lipídeos e exopolissacarídeos. PPG em Engenharia de Pesca, UFC.

28. Mayra Bezerra Vettorazzi - doutorado, 2018. Parâmetros seminais e proteoma do sêmen de ariacó (Lutjanus synagris, Perciformes) tratados com gonadotropina coriônica humana (HCG). PPG em Ciências Marinhas Tropicais, UFC.

29. Virna Mirela Maciel Silva - mestrado, 2018. Caracterização da população de Hippocampus reidi no estuário do rio Pacoti, Ceará. PPG em Engenharia de Pesca, UFC. 
30. Sara Andrade dos Santos - mestrado, 2018. Diagnóstico socioeconômico dos pescadores artesanais de lagosta em Fortaleza, CE. PPG em Engenharia de Pesca, UFC.

31. Márcia Darlene Pereira Santana - mestrado, 2018. Produção integrada de peixes e vegetais, em sistema de recirculação de água, com aproveitamento do efluente. PPG em Engenharia de Pesca, UFC.

32. Manoela Wariss Figueiredo - doutorado, 2018. Uso e conservação de recursos pesqueiros em unidades de conservação na bacia do médio rio Xingu, Pará, Brasil. PPG em Engenharia de Pesca, UFC.

33. Caio de Góes Loira Parada - mestrado, 2019. Análise da pesca de pequena escala dos elasmobrânquios desembarcados em Paracuru, Ceará. PPG em Engenharia de Pesca, UFC.

34. João Henrique Cavalcante Bezerra - doutorado, 2020. Cultivo de microalga: extração de biocompostos e sua administração a camarões Penaeus vannamei. PPG em Ciências Marinhas Tropicais, UFC.

35. Lucas Daniel Borges - mestrado*, 2020. Caracterização microbiológica de peixes ornamentais recifais. PPG em Engenharia de Pesca, UFC. *Coorientador.

\section{SUPERVISÃO DE PESQUISADORES-DOUTORES}

1. José Renato de Oliveira César - Desenvolvimento Científico Regional (2007-2009), Conselho Nacional de Desenvolvimento Científico (CNPq)/ Fundação Cearense de Apoio ao Desenvolvimento Científico e Tecnológico (Funcap). Departamento de Engenharia de Pesca, UFC.

2. Vicente Vieira Faria - Desenvolvimento Científico Regional CNPq/Funcap (2007-2010) e Pesquisador Colaborador Funcap (2010-2011). Instituto de Ciências do Mar (Labomar), UFC.

3. Raúl Cruz Izquierdo - Pesquisador Visitante Funcap (2009-2010) e Pesquisador Visitante CNPq (2010-2012). Labomar, UFC.

\section{TÍTULOS E HOMENAGENS}

1998-1999 Fellow of the School of Graduate Studies; Memorial University of Newfoundland, Canadá. https://www.mun.ca/sgs/current/scholarships/fellows_1998_1999.php.

2004 "Personalidade da Pesca"; Conselho Regional de Engenharia e Arquitetura (CREA/CE).

2010 “50 anos do Labomar/UFC" (na época diretor do Labomar); Assembleia Legislativa do Estado do Ceará.

2011 “Amigo do Peixe"; jornal O Povo, Ceará.

2013 “Comenda Terezinha Martins" (personalidade cearense); jornal O Estado, Ceará.

2014 "Destaque Político" do Dia do Engenheiro de Pesca"; Assembleia Legislativa do Estado do Ceará e Associação dos Engenheiros de Pesca do Estado do Ceará.

2014 “Troféu Estrela do Mar" (na época secretário adjunto de Pesca e Aquicultura do Ceará); Fenacam - Feira Nacional do Camarão.

2015 "Troféu Santa Tilápia" (homenagem a personalidades que se destacaram na tilapicultura); Festival da Tilápia, Ceará. 
2017 Homenagem da União Brasileira dos Estudantes Secundaristas (Ubes).

2017 Homenagem da 10a Bienal da União Nacional dos Estudantes (UNE).

2019 Homenagem pelos serviços prestados como pró-reitor na gestão 2015-2019; PróReitoria de Assuntos Estudantis da UFC.

\section{HOMENAGENS PÓSTUMAS}

A Sociedade Brasileira para o Estudo de Elasmobrânquios (SBEEL), a Sociedade Latino Americana de Especialistas em Mamíferos Aquáticos (Solamac) e a Associação de Pesquisa e Preservação de Ecossistemas Aquáticos (Aquasis) emitiram nota de pesar.

O Departamento de Engenharia de Pesca, o Departamento de Biologia, o Instituto de Ciências do Mar (Labomar) e a Pró-Reitoria de Assuntos Estudantis, todos da UFC, decretaram luto oficial de três dias.

O falecimento de Manuel Furtado Neto coincidiu com a data em que se comemora o Dia Nacional do Engenheiro de Pesca, 14 de dezembro. Durante sessão solene realizada em alusão ao Dia Nacional do Engenheiro de Pesca, na Assembleia Legislativa do Estado do Ceará, em 16 de dezembro de 2019, a Associação dos Engenheiros de Pesca do Estado do Ceará e a Assembleia Legislativa do Estado do Ceará homenagearam o professor Manuel na forma de um minuto de silêncio (com emocionante sequência de imagens exibidas em um telão mostrando o Manuel engenheiro, professor, filho, marido, pai e amigo).

\section{AGRADECIMENTOS}

Os autores agradecem:

(1) Ana Caroline M. V. de Andrade Furtado (Carol), esposa do professor Manuel Furtado Neto, pelo acesso a documentos e pelas informações essenciais para a construção do presente texto;

(2) Bartolomeu W. S. de Souza e Francisca Gleire R. de Menezes (coordenador e membro da coordenação, respectivamente, do Programa de Pós-Graduação em Engenharia de Pesca da UFC), Alessandra Cristina da Silva-Farias (coordenadora do curso de Engenharia de Pesca da UFC), Érika F. Mota e Pablo R. da Silva (coordenadora e secretário, respectivamente, do curso de graduação em Ciências Biológicas, bacharelado e licenciatura, da UFC) e Ana Carolina O. de Meirelles (Aquasis), pelas informações sobre as monografias, dissertações e teses;

(3) Killian Heffernan e Raúl Cruz Izquierdo, pelas informações sobre vínculos de pesquisa com o Manuel;

(4) Ricardo Toniolli, editor-chefe da Ciência Animal, pela atenção quanto aos questionamentos relativos às publicações de Manuel neste periódico;

(5) Patricia N. Bordallo, Ana Carolina O. de Meirelles, Patricia Charvet e Maria Eliete V. Faria, pela revisão, pelos comentários e pelas sugestões no manuscrito;

(6) Cristina A. Rocha Barreira, editora-chefe da revista Arquivos de Ciências do Mar, pelo honroso convite para a redação desta homenagem ao inestimável professor Manuel A. A. Furtado Neto. 